\title{
(Neu)Bewertung und Eingruppierung von Facharbeit in der chemischen Industrie und in der Metall- und Elektroindustrie Baden-Württembergs
}

Facharbeit ist der traditionelle Bezugspunkt in der Arbeitsbewertung und in der tariflichen Entgeltpolitik. Im Kontext der Reform der Entgelttarifrahmenverträge wurde dieser Eckpunkt verschoben. Facharbeit wurde im Vergleich zu anderen Qualifikationsniveaus aufgewertet und die beruflichen Entwicklungsmöglichkeiten von gewerblich Beschäftigten wurden tariftechnisch durch Beseitigung der Statusdifferenzierung zwischen Arbeitern und Angestellten erweitert. Der Beitrag beschreibt, welche Zielsetzungen die Entgeltreformprojekte hatten, wie Facharbeit vor und nach der Tarifreform definiert, bewertet und eingruppiert ist, ob die intendierten Ziele erreicht wurden und welcher Preis dafür bezahlt wurde.

REINHARD BAHNMÜLLER

\section{Zielsetzungen der Entgeltreform- projekte}

Eine Reform der Lohn- und Gehaltsrahmenabkommen stand schon seit den frühen 1980er Jahren auf der tarifpolitischen Tagesordnung, ihre Durchsetzung gestaltete sich jedoch ausgesprochen langwierig und zäh. In der chemischen Industrie wurde 1987 der erste Abschluss erreicht, in der Metall- und Elektro $(\mathrm{M}+\mathrm{E})$-Industrie dauerte es 15 Jahre länger, in anderen Branchen wurde die Reform bisher nur in Teilen abgeschlossen (z. B. Banken, öffentlicher Dienst) oder die Gespräche zwischen den Tarifparteien sind noch im Gange (z. B. Einzelhandel, Textil- und Bekleidungsindustrie). Die Zielsetzungen der Entgeltreformprojekte waren und sind bei allen Unterschieden im Einzelnen in ihrem Kern dieselben. Es ging und es geht um:

- die Realisierung einer einheitlichen Arbeitsbewertung für Arbeiter, Kaufleute, Techniker und Meister auf der Basis eines für alle gleichen Systems,

- die Beseitigung der Ungleichbehandlungen von Arbeitern und Angestellten sowie die gerechte Bewertung von Arbeit und Leistung innerhalb dieser Statusgruppen, die relative Aufwertung von gewerblicher und teilweise auch kaufmännischer Facharbeit und ihre Gleichstellung mit technischen Angestellten, die Arbeitsaufgaben mit vergleichbaren Anforderungen zu bewältigen haben.
Zu einem übergeordneten Ziel der Reformprojekte entwickelte sich zudem die Stabilisierung des Systems der Flächentarifverträge (Brunkhorst et al. 2006). In den 1980er Jahren war dieses Ziel noch nebensächlich, es rückte allerdings im Laufe der Zeit immer mehr in den Vordergrund und wurde schließlich in der M+E-Industrie derart bedeutsam, dass der Fortbestand des Tarifsystems arbeitgeberseitig an den Abschluss der Entgeltrahmenabkommen (ERA) gebunden wurde. ERA entwickelte sich somit von einem Gleichstellungs- bzw. Emanzipationsprojekt der Arbeiterschaft im Verhältnis zu den Angestellten zu einem Stabilisierungsprojekt des Flächentarifvertrags (Bahnmüller/Schmidt 2009).

Arbeitgeber wie Gewerkschaften knüpften die Tarifreform an Bedingungen. Die Arbeitgeber verlangten Kostenneutralität, d.h. durch die Veränderung der Wertigkeitsrelationen von Tätigkeiten und die dadurch ausgelösten Entgelteffekte sollten die Verteilungsrelationen zwischen Kapital und Arbeit nicht zu ihren Ungunsten verschoben werden. Die Gewerkschaften wiederum wollten die Beschäftigten vor den Folgen möglicher Abgruppierungen schützen und verlangten eine Verdienstabsicherung für Altbeschäftigte.

Die Aufwertung von Facharbeit war auch in den beiden hier untersuchten Entgeltreformprojekten - der Bundesentgeltrahmentarifvertrag (BETV) für die chemische Industrie und das Entgeltrahmenabkommen (ERA) für die $\mathrm{M}+\mathrm{E}$-Industrie - ein zentrales Ziel. Besser bewertet 
und bezahlt werden sollte vor allem gewerbliche Facharbeit, deren Attraktivität nach Einschätzung beider Tarifparteien der M + E-Industrie dadurch gelitten hatte, dass die Verdienste von Un- und Angelernten im Leistungslohn nicht selten über jenen von Facharbeitern im Zeitlohn lagen. ${ }^{1}$ Materielle Anreize zur (Höher)Qualifizierung waren somit aus Sicht der Tarifparteien nur noch unzureichend gegeben. Dem sollte durch eine größere Spreizung im unteren Entgeltsegment bzw. durch eine finanzielle Aufwertung von Facharbeit entgegengewirkt werden.

Unterbewertet war gewerbliche Facharbeit jedoch vor allem im Vergleich zu jener von technischen Angestellten, die vergleichbare Arbeitsanforderungen zu bewältigen hatten. So lag das Grundentgelt der Facharbeiter-Ecklohngruppe (LG 7) im Zeitlohn der M+E-Industrie Baden-Württembergs (BaWü) vor der Einführung des ERA um mehr als $400 €$ unter der als gleichwertig geltenden Tarifgruppe der Techniker (T 2/4) - und der Abstand wuchs jährlich. Auch Kaufleute verdienten weniger als Techniker. Erklärte Absicht der Tarifparteien war es deshalb, die Entgeltlinie für gewerbliche Facharbeit und jene für Kaufleute an die von Technikern anzugleichen. Bei der Beseitigung der Statusunterschiede zwischen Arbeitern und Angestellten und der Aufwertung von Facharbeit spielten in der $\mathrm{M}+\mathrm{E}$-Industrie nicht zuletzt strategische Überlegungen eine Rolle: Den absehbaren Schwierigkeiten, auch künftig Jugendliche für eine Berufsausbildung im dualen System zu gewinnen, sollte offensiv durch eine Egalisierung der Vergütung sowie eine Erweiterung der beruflichen Entwicklungschancen begegnet werden.

$\mathrm{Ob}$ auch in der chemischen Industrie, in der die Tarifreform bereits 15 Jahre zuvor realisiert worden war, solche Überlegungen mitspielten, ist fraglich, da Fachkräftemangel seinerzeit noch kein Thema war. Aber auch hier gab es eine ungleiche Bezahlung gleichrangiger Arbeit, was zunehmend als dysfunktional angesehen wurde und bei (gewerblich) Beschäftigten zu Unzufriedenheit führte. Angestellte verdienten zwar am Anfang ihres Berufsweges (zum Teil erheblich) weniger als Facharbeiter, ließen jedoch die Arbeiter nach zwei bis sechs Jahren hinter sich und verdienten in der Endstufe entschieden mehr als diese. So kam ein Handwerker mit abgeschlossener Berufsausbildung nach sechs Jahren auf 2.638 DM im Monat, ein technischer Angestellter mit entsprechender Ausbildung demgegenüber auf 3.609 DM (Kädtler 2002, S. 2). Unterschiedlich waren - mit Ausnahme der untersten Gehaltsgruppe - auch die Verdienste zwischen den beiden Angestelltengruppen. Anders als in der M + EIndustrie verdienten in der chemischen Industrie allerdings kaufmännische Angestellte mit abgeschlossener Berufsausbildung und sechsjähriger Berufserfahrung 443 DM mehr als technische. Diese beiden Einkommensungerechtigkeiten galt es nach dem Willen der Tarifpolitiker zu beseitigen.

\section{Die Zentralität von Facharbeit und Methoden der Arbeitsbewertung}

Facharbeit kommt in der Arbeitsbewertung, die der Eingruppierung in Entgeltgruppen zugrunde liegt, eine Schlüsselfunktion zu. Durch sie wird die Eckentgelt- oder 100 \%-Gruppe definiert, in die Tätigkeiten eingruppiert sind, die eine abgeschlossene berufliche Ausbildung verlangen. Die Eckentgeltgruppe markiert die traditionelle Trennlinie von gelernter und ungelernter Arbeit. Stellvertretend für andere Tarifgruppen ist sie Verhandlungsgegenstand zwischen den Tarifparteien, wenn es um Entgelterhöhungen oder die Berechnung von Zulagen aller Art geht, und nicht zuletzt ist sie der Vermessungspunkt für das Ausmaß an Entgeltspreizung und -differenzierung, das in einer Branche gelten soll.

Welchen Stellenwert das Ausbildungsniveau und damit auch „Facharbeit“ für die Eingruppierung und Bezahlung hat, ist von der eingesetzten Methode der Arbeitsbewertung abhängig. Dabei konkurrieren traditionell unterschiedliche Ansätze, denen divergierende Gerechtigkeitsvorstellungen zugrunde liegen. Auf der einen Seite stehen personen- bzw. bedarfsorientierte Bewertungsansätze, auf der anderen anforderungsorientierte. Personen- und bedarfsorientierte Ansätze berücksichtigen Aspekte sozialer Gerechtigkeit und individuelle Fähigkeiten, etwa das Alter, den Familienstand oder auch die „mitgebrachte“ individuelle Qualifikation. Eine qualifikationsorientierte Bezahlung war in der Industrie und im Handwerk noch bis in die 1930er Jahre vorherrschend (Meine et al. 2011, S. 134). Mittlerweile ist sie von der anforderungsorientierten Arbeitsbewertung verdrängt worden. Bei diesem Ansatz steht nicht eine konkrete Person mitsamt ihren Kompetenzen und sozialen Bezügen im Mittelpunkt der Bewertung, sondern die Komplexität der Arbeitsaufgabe und die daraus resultierenden Arbeitsanforderungen. Für den Umgang mit „Qualifikation“ bedeutet dies: formale Ausbildungsabschlüsse, über die Beschäftigte verfügen (z. B. Schul-, Hochschul- oder Berufsabschlüsse), sind für die Eingruppierung nicht ausschlaggebend. Bewertet wird ausschließlich die Arbeitsaufgabe. Verlangt deren Bewältigung ein bestimmtes Qualifikationsniveau, ist für die Eingruppierung nur bedeutsam, ob dieses bei der Person, der die Arbeitsaufgabe übertragen wird, vorhanden ist, nicht auf welche Art und Weise es erworben wurde. Entsprechend heißt es im BETV für die chemische Industrie: „Die Arbeitnehmer werden entsprechend der von ihnen ausgeübten Tätigkeit in die Entgeltgruppen eingruppiert. Für die Eingruppierung in eine Entgeltgruppe ist nicht die beruf-

1 Beschäftigte im Leistungslohn (Akkord/Prämie) erhielten vor ERA betriebsdurchschnittlich mindestens $30 \%$ Leistungszulage, im Zeitlohn wurden dagegen nur $15 \%$ bezahlt. Mit dem ERA wurden die Leistungsentgeltanteile für alle Beschäftigten auf $15 \%$ egalisiert. 
liche Bezeichnung, sondern allein die Tätigkeit des Arbeitnehmers maßgebend" ( $\$ 2$ BETV Chemie).

Die Beschreibung der qualifikatorischen Anforderungen, die für die Ausführung der Tätigkeiten erforderlich sind, erfolgt gleichwohl auch in anforderungsorientierten Bewertungssystemen, wie sie in den beiden hier untersuchten Branchen Anwendung finden, durch einen Verweis auf schulische oder berufliche Ausbildungsniveaus (Übersicht 1). Auch wirken Ausbildungsabschlüsse de facto nicht selten als "gläserne Deckel“ in der Eingruppierung (s. u.).

Die Qualifikation, genauer gesagt die „Qualifikationsanforderungen“, sind in den verschiedenen Varianten anforderungsbezogener Entlohnung das wichtigste, jedoch nicht das einzige Bewertungsmerkmal. Neben qualifikationsbezogenen Bewertungsmerkmalen kommen auch verantwortungsbezogene („Verantwortung für die eigene $\mathrm{Ar}$ beit“ bzw. „für die Arbeit und die Sicherheit anderer“) und belastungs- bzw. umweltbezogene Kriterien zur Anwendung (Lärm, Schmutz etc.). In analytischen Systemen wird jedes dieser Merkmale für sich bewertet, gewichtet, bepunktet und in einem Arbeitswert zusammengefasst, der einer Arbeitswert- bzw. Lohngruppe entspricht. In summarischen Systemen erfolgt die Bewertung der Arbeitsaufgabe in einer Gesamtbetrachtung. Summarische Systeme sind weiter verbreitet als analytische. Daran haben auch die Entgeltreformprojekte der letzten Jahre nichts geändert.

\section{Die Entgeltsysteme und das Spektrum von „Facharbeit" im BETV und im ERA Baden-Württembergs}

In der chemischen Industrie erfolgt die Eingruppierung vor wie nach dem BETV - auf Basis eines summarischen Systems. Grundlage der Eingruppierung sind die in den Entgeltgruppenbeschreibungen enthaltenen Oberbegriffe. Als Erläuterung können die bei den Entgeltgruppenbeschreibungen aufgeführten „Richtbeispiele“ bzw. Berufe herangezogen werden (Übersicht 1). Passen die Oberbegriffe nicht auf eine ausgeübte Tätigkeit, „so ist ein Arbeitnehmer in diejenige Entgeltgruppe einzugruppieren, die seiner Tätigkeit am nächsten kommt“ (BETV \$3). Werden mehrere Tätigkeiten ausgeübt, auf die verschiedene Entgeltgruppen zutreffen, ist in jene Entgeltgruppe einzugruppieren, „deren Anforderungen den Charakter seines Arbeitsbereichs im Wesentlichen bestimmen.“

Summarische Systeme der Arbeitsbewertung sind auch in der M + E-Industrie vorherrschend (Ehlscheid et al. 2006, S. 123). Einen Sonderweg gingen Baden-Württemberg und Nordrhein-Westfalen. Hier kreierten die Tarifparteien im Kontext der ERA-Reform ein neues System, eine Synthese aus dem bisherigen analytischen und dem summarischen Verfahren. Das sogenannte Stufenwertzahlverfahren arbei- tet mit fünf Bewertungsmerkmalen: (1) „Wissen und Können“, (2) „Denken“, (3) „Handlungsspielraum/Verantwortung“, (4) „Kommunikation“ und (5) „Mitarbeiterführung“. Die Differenzierung innerhalb der Bewertungsmerkmale erfolgt auf der Grundlage summarisch beschriebener Stufen. Jedem Merkmal bzw. jeder Stufe innerhalb des Merkmals ist eine Wertzahl (bzw. sind Punkte) zugeordnet. Aus der Summe der Punktwerte ergibt sich die Entgeltgruppe. Maximal lassen sich 96 Punkte erzielen. Am höchsten gewichtet ist das Bewertungsmerkmal „Wissen und Können“ (41\%), am niedrigsten „Mitarbeiterführung“ (7\%). Zwei der Bewertungsmerkmale, die nunmehr Anwendung finden, waren im bisherigen (analytischen) Arbeitsbewertungssystem nicht enthalten: „Handlungsspielraum“ und „Kommunikation".

Das Anforderungsniveau von Facharbeit markiert in summarischen wie in analytischen Systemen die Scheidelinie zwischen gelernter und ungelernter Arbeit. Wo ungelernte Arbeit endet und gelernte beginnt, ist jedoch umstritten. Seinen Niederschlag hat dieser Streit auch in den reformierten Entgeltrahmentarifverträgen gefunden. Sowohl im BETV als auch in den ERA-TVs wurde auf Druck der Arbeitgeber unterhalb der Eckentgeltgruppe, die ein Qualifikationsniveau vorsieht, wie es i.d. R. durch eine dreibis dreieinhalbjährige Berufsausbildung erworben wird, eine zweijährige Berufsausbildung aufgenommen. Sie ist ein bis zwei Entgeltgruppen unterhalb der traditionellen Eckentgeltgruppe eingruppiert (Übersicht 1 und 2). Die Untergrenze von Facharbeit lässt sich deshalb nun sowohl auf dem Niveau einer mindestens dreijährigen als auch einer zweijährigen Berufsausbildung ziehen. In der betrieblichen Praxis ist die Relevanz der verkürzten Berufsausbildung bisher allerdings noch marginal.

Nach oben lässt sich das auf einer dualen Berufsausbildung basierende Segment der Facharbeit ebenfalls in doppelter Weise abgrenzen. In einem engen Verständnis liegt die Obergrenze bei einem Anforderungsniveau, bei dem neben einer Berufsausbildung keine Zusatzausbildung bzw. Aufstiegsfortbildung (z. B. IHK-Meister, Techniker) vorausgesetzt wird. Man kann das Segment von Facharbeit allerdings auch weiter fassen und die Obergrenze erst dort ziehen, wo es um Arbeitsaufgaben auf einem Qualifikationsniveau geht, für das i.d.R. ein abgeschlossenes Fachhochschulstudium notwendig ist. Hier beginnt das Segment von Arbeitsaufgaben, das i.d. R. von Hochschulabsolventen besetzt wird. ${ }^{2}$ Es umfasst auch das sich in den letzten Jahren entwickelnde und auf einem dualen Studium basierende akademische Seg-

2 Das akademische Segment ist mittlerweile Beschäftigten ohne Abitur oder Fachhochschulreife nicht mehr grundsätzlich verschlossen. So wurde etwa im Jahr 2010 am Steinbeis Center forTechnology mit dem „Bachelor of Arts in Business Administration“ ein Studiengang eingerichtet, der erfahrenen Praktikern aus verschiedenen Unternehmensbereichen offensteht. 


\section{Entgeltgruppenbeschreibungen und Richtbeispiele in der chemischen Industrie}

\begin{tabular}{|c|c|c|c|}
\hline & & Beschreibung (Kurzfassung) & Beruf (Beispiele) \\
\hline & EG 1 & Tätigkeiten, die eine kurze Einweisung erfordern: & Bote \\
\hline & EG 2 & $\begin{array}{l}\text { Tätigkeiten, für die Kenntnisse und Fertigkeiten erforderlich sind, } \\
\text { die durch eine Berufspraxis von i.d.R. bis zu } 13 \text { Wochen erworben werden. }\end{array}$ & \\
\hline & EG 3 & $\begin{array}{l}\text { Tätigkeiten, für die Kenntnisse und Fertigkeiten erforderlich sind, } \\
\text { die durch eine Berufspraxis von i.d.R. } 6 \text { bis } 12 \text { Monaten erworben werden. }\end{array}$ & \\
\hline $\mathbf{F}$ & EG 4 & $\begin{array}{l}\text { Tätigkeiten, für die Kenntnisse und Fertigkeiten erforderlich sind, } \\
\text { die durch eine abgeschlossene zweijährige Berufsausbildung erworben worden sind } \\
\text { + eingehende Anweisungen. }\end{array}$ & $\begin{array}{l}\text { Chemiebetriebswerker/in, } \\
\text { Chemielaborwerker/in, } \\
\text { Elektroanlageninstallateur/in }\end{array}$ \\
\hline A & EG 5 & $\begin{array}{l}\text { Tätigkeiten, für die Kenntnisse und Fertigkeiten erforderlich sind, } \\
\text { die über die Anforderungsmerkmale der Gruppe E } 4 \text { hinausgehen. }\end{array}$ & \\
\hline C & EG 6 & $\begin{array}{l}\text { Tätigkeiten, für die Kenntnisse und Fertigkeiten erforderlich sind, } \\
\text { die durch eine abgeschlossene mindestens dreijährige Berufsausbildung erworben werden. }\end{array}$ & $\begin{array}{l}\text { Kaufmann/-frau, } \\
\text { Chemokant/in, Pharmakant/in, } \\
\text { Technische Zeichner/in, } \\
\text { Fachkraft für Lagerwirtschaft }\end{array}$ \\
\hline A & EG 7 & $\begin{array}{l}\text { Tätigkeiten, für die Kenntnisse und Fertigkeiten erforderlich sind, die über die } \\
\text { Anforderungsmerkmale der Gruppe EG } 6 \text { hinausgehen und einen größeren } \\
\text { Abstraktionsgrad der Lerninhalte aufweisen (mindestens eine Fremdsprache). }\end{array}$ & $\begin{array}{l}\text { Chemielaborant/in, } \\
\text { Prozessleitelektroniker/in, } \\
\text { Meister mit einem } \\
\text { einfachen Arbeitsgebiet }\end{array}$ \\
\hline $\mathbf{R}$ & EG 8 & $\begin{array}{l}\text { Schwierige Spezialtätigkeiten, für die Kenntnisse und Fertigkeiten erforderlich sind, } \\
\text { die über die Anforderungsmerkmale der Gruppe E } 7 \text { hinausgehen } \\
\text { + planmäßige betriebliche Spezialausbildung oder längere Berufserfahrungen. }\end{array}$ & \\
\hline \multirow[t]{2}{*}{$\mathbf{E}$} & EG 9 & $\begin{array}{l}\text { Höherwertige kaufmännische oder technische Tätigkeiten, für die Kenntnisse und Fertigkeiten } \\
\text { erforderlich sind, die eine abgeschlossene mindestens dreijährige Berufsausbildung } \\
+ \text { eine abgeschlossene funktionsbezogene zusätzliche Aus- oder Weiterbildung oder } \\
\text { zusätzliche Fachkenntnisse erfordern. }\end{array}$ & \\
\hline & EG 10 & $\begin{array}{l}\text { Hochwertige kaufmännische oder technische Tätigkeiten, für die Kenntnisse und Fertigkeiten } \\
\text { erforderlich sind, die eine abgeschlossene mindestens dreijährige Berufsausbildung } \\
\text { + eine Zusatzausbildung zum Chemotechniker/staatlich anerkannterTechniker oder eine } \\
\text { vergleichbare kaufmännische Zusatzausbildung erfordern. }\end{array}$ & $\begin{array}{l}\text { Chemotechniker/in, } \\
\text { Techniker/in }\end{array}$ \\
\hline & EG 11 & $\begin{array}{l}\text { Selbstständige kaufmännische oder technischeTätigkeiten, für die Kenntnisse und Fertigkeiten } \\
\text { erforderlich sind, die eine FH-Ausbildung oder einen gleichwertigen Abschluss erfordern. }\end{array}$ & Betriebswirt/in, Ingenieur/in \\
\hline & EG 12 & $\begin{array}{l}\text { Selbstständige kaufmännische oder technischeTätigkeiten, für die Kenntnisse und Fertigkeiten } \\
\text { erforderlich sind, die eine FH-Ausbildung + Spezialwissen + Berufserfahrungen erfordern. }\end{array}$ & \\
\hline & EG 13 & $\begin{array}{l}\text { Tätigkeiten, für die Kenntnisse und Fertigkeiten erforderlich sind, die eine FH-Ausbildung + } \\
\text { Spezialwissen + Berufserfahrungen + Leitungsaufgaben + Verantwortung für Teilgebiete erfordern. }\end{array}$ & \\
\hline
\end{tabular}

ment von Facharbeit, das hier jedoch außer Betracht bleibt (zum dualen Studium siehe den Beitrag von Krone/Mill in diesem Heft). Bei der engen wie der weiten Variante der Grenzziehung von Facharbeit muss allerdings in Rechnung gestellt werden, dass sich in den jeweiligen Entgeltkorridoren de facto auch Beschäftigte ohne abgeschlossene Berufsausbildung bzw. Zusatzausbildung befinden können, da, worauf bereits hingewiesen wurde, in die anforderungsbasierte Bewertung von Arbeit neben der Qualifikation auch andere Aspekte eingehen. Entgeltgruppen für Facharbeit werden somit auch von Beschäftigten erreicht, die keinen einschlägigen berufsqualifizierenden Abschluss haben. ${ }^{3}$
Subsumiert man unter Facharbeit solche Tätigkeiten, die entsprechend der traditionellen Definition der Untergrenze eine mindestens dreijährige Berufsausbildung, jedoch keine Zusatzausbildung bzw. Aufstiegsfortbildung verlangen (z. B. Meister, Techniker, Fachwirte), um-

3 Im Jahr 2005 waren ca. drei Viertel der in der M+E-Industrie Baden-Württembergs beschäftigten Un- und Angelernten auf einem Eingruppierungsniveau, das einem berufsqualifizierenden Abschluss entspricht oder noch höher liegt (vgl. Bahnmüller/Schmidt 2009, S. 71). 
ÜBERSICHT 2

Ausbildungsstufen und Entgeltspektrum unter- und oberhalb der Eckenentgeltgruppe im ERA Baden-Württembergs

\begin{tabular}{|c|c|c|}
\hline \multicolumn{3}{|c|}{ Anlernen } \\
\hline Stufe & Begründung/Kriterium & Entgeltspektrum \\
\hline A 1 & Einmalige Arbeitsunterweisung und kurze Übung & EG 1 \\
\hline A 2 & Arbeitsunterweisung und längere Übung & EG 2 \\
\hline A 3 & $\begin{array}{l}\text { Arbeitsunterweisung und Übung } \\
\text { über mehrere Wochen }\end{array}$ & EG 2-4 \\
\hline A 4 & $\begin{array}{l}\text { Systematisches Anlernen inkl. } \\
\text { theoretischer Kenntnisse bis zu } 1 / 2 \text { Jahr }\end{array}$ & EG 3-5 \\
\hline A 5 & $\begin{array}{l}\text { Umfangreiches systematisches Anlernen } \\
\text { über ein halbes Jahr hinaus }\end{array}$ & EG 5-6 \\
\hline \multicolumn{3}{|c|}{ Berufsausbildung } \\
\hline Stufe & Begründung/Kriterium & Entgeltspektrum \\
\hline B 1 & Abgeschlossene Berufsausbildung 2 Jahre & EG 5-6 \\
\hline B 2 & Abgeschlossene Berufsausbildung 3-3,5 Jahre & EG 7-10 \\
\hline B 3 & $\begin{array}{l}\text { Abgeschlossene Berufsausbildung + Fachaus- } \\
\text { bildung } 1 \text { Jahr (z. B. Meister-Ausbildung IHK) }\end{array}$ & EG $10-14$ \\
\hline B 4 & $\begin{array}{l}\text { Abgeschlossene Berufsausbildung + Fachaus- } \\
\text { bildung } 2 \text { Jahre (z. B. staatlich geprüfterTechniker) }\end{array}$ & EG $11-15$ \\
\hline B 5 & Abgeschlossenes Fachhochschulstudium & EG $12-17$ \\
\hline B 6 & Abgeschlossenes Universitätsstudium & EG 13-17 \\
\hline
\end{tabular}

fasst dieses Segment im ERA Baden-Württembergs vier Entgeltgruppen (EG 7 - 10). Die Entgeltspanne reicht von $2.712 €$ (EG 7) bis $3.295 €$ (EG 10) (Stand 5/2012, ohne Leistungsentgeltanteil). Im BETV der chemischen Industrie ist der Entgeltkorridor für Facharbeit ohne eine weitere Zusatzqualifikation etwas enger. Hierfür stehen die Entgeltgruppen 6 bis 8 zur Verfügung, ggf. könnte noch die EG 9 hinzugezählt werden, die allerdings bereits eine „abgeschlossene funktionsbezogene zusätzliche Aus- oder Weiterbildung“ oder „zusätzliche Fachkenntnisse“ verlangt. Ab EG 10 wird eine Zusatzausbildung zum Chemotechniker, zum staatlich anerkannten Techniker oder eine vergleichbare kaufmännische Zusatzausbildung vorausgesetzt. Die Entgeltspanne reicht in der engen Definition von $2.602 €$ (EG 6, Eingangsstufe) bis $3.348 €$ (EG 8), in der weiteren bis $3.530 €$ (EG 9) (jeweils Endstufe; Werte für Hessen, Stand 7/2012). Die Eckentgelte beider Branchen unterscheiden sich nicht unerheblich. Berücksichtigt man die allen Beschäftigten der $\mathrm{M}+\mathrm{E}-$ Industrie zustehende tarifliche Leistungszulage von betriebsdurchschnittlich $15 \%$, liegt das Eckentgelt in der
M + E-Industrie BaWü $412 €(17 \%)$ über dem Niveau der chemischen Industrie, was verdeutlicht, dass Facharbeit selbst in zwei Branchen mit starken Gewerkschaften und noch relativ hoher Tarifbindung sehr unterschiedlich bezahlt wird. ${ }^{4}$

Beziehen wir zur Bestimmung der beruflichen und entgeltlichen Entwicklungschancen von Facharbeit in einem zweiten Schritt jene Beschäftigten mit ein, die zusätzlich zu einer mindestens dreijährigen Berufsausbildung eine einoder zweijährige Zusatzausbildung bzw. Aufstiegsfortbildung vorweisen können, erweitert sich das Eingruppierungsspektrum in der chemischen Industrie um eine, in der $\mathrm{M}+\mathrm{E}-$-Industrie BaWü um bis zu fünf Entgeltgruppen. In der chemischen Industrie stehen ihnen somit zumindest formal insgesamt fünf, in der $\mathrm{M}+\mathrm{E}$-Industrie BaWü neun Entgeltgruppen offen. In der chemischen Industrie können sie maximal die EG 10 (von 13), in der M+E-Industrie BaWü die EG 15 (von 17) erreichen.

\section{Die Eingruppierung von Facharbeit vor und nach den Entgeltreformen}

Wurde bisher die in den Tarifverträgen vorgesehene Methodik und Systematik der Eingruppierung behandelt, wenden wir uns nun der tatsächlichen Eingruppierung in den beiden Branchen zu. Dazu stellen wir im ersten Schritt die Dynamik der Eingruppierung vor den Tarifreformprojekten dar (4.1). Im zweiten Schritt gehen wir der Frage nach, ob die intendierten Reformziele erreicht wurden und wer die Gewinner bzw. Verlierer der Reform waren (4.2). Abschnitt 4.3 untersucht, wie sich die Eingruppierung im Nachhinein entwickelt hat.

\subsection{Eingruppierung vor den Entgeltreformpro- jekten}

Vor Einführung des BETV und des ERA war der Entwicklungskorridor, der Arbeitern und Angestellten offenstand, durch die gruppenspezifischen Systeme für Arbeiter, Kaufleute, Techniker und Meister vorgegeben - und er war nicht zu überspringen. Für gewerbliche Facharbeit in der M +EIndustrie BaWü gab es im alten Lohnsystem sieben Arbeitswert- bzw. Lohngruppen, für jene der chemischen Industrie dagegen nur zwei. Bei den Angestellten lässt sich das den Fachangestellten vorbehaltene Segment nicht so eindeutig bestimmen, da die Grenzen nach „oben“ fließend bzw. nicht

4 Aber auch innerhalb der jeweiligen Branchen gibt es regional erhebliche Unterschiede.

5 Zwar war in der $\mathrm{M}+\mathrm{E}$-Industrie $\mathrm{BaWü} \mathrm{in} \mathrm{der} \mathrm{Entgeltgrup-}$ penbeschreibung T 4 für technische Angestellte als Ausbil- 
vorhanden waren. ${ }^{5}$ Tarifsystematisch war die Durchlässigkeit bei Angestellten mit qualifizierter Berufsausbildung somit tarifsystematisch auch schon vor der Reform der Entgeltrahmentarifverträge gegeben. ${ }^{6}$ In der $\mathrm{M}+\mathrm{E}$-Industrie BaWü standen Angestellten mit abgeschlossener Berufsausbildung prinzipiell sechs, in der chemischen Industrie fünf Entgeltgruppen zur Verfügung. Deutliche Unterschiede gab es jedoch in der Anzahl der Gehaltsgruppen unter der Eckentgeltgruppe: in der M+E-Industrie BaWü existierte für Angestellte nur eine, in der chemischen Industrie waren es dagegen drei.

Wie entwickelte sich nun die Besetzung der Lohn- und Gehaltsgruppen? Die Richtung war in beiden Branchen und auch bezogen auf beide Statusgruppen - allerdings mit unterschiedlich starker Ausprägung - dieselbe: Der Anteil der Beschäftigten unterhalb der Ecklohngruppe (mindestens dreijährige Berufsausbildung) nahm kontinuierlich ab, jener von Facharbeit nahm zu. In der M+E-Industrie BadenWürttembergs verringerte sich der Anteil unterhalb der Ecklohngruppe im Zeitraum von 1977 bis 2005 um die Hälfte. Was die unteren Arbeitswert- bzw. Lohngruppen verloren, gewannen die oberen hinzu, wobei die „Einsteigergruppen“ von Facharbeit (AW 6-8) im Vergleich zum oberen Entlohnungssegment vergleichsweise wenig $\mathrm{Zu}$ wachs zu verzeichnen hatten (Tabelle 1).

Eine ähnliche Entwicklung wie im gewerblichen Bereich zeigt sich bei den kaufmännischen Angestellten: Der Anteil der Beschäftigten in den unteren Entgeltgruppen K1 und $\mathrm{K} 2 \mathrm{nahm}$ ab, in den mittleren Gruppen blieb die Besetzung in etwa stabil (K 3-4), und in der "Oberliga“ gab es beträchtliche Zuwächse. Noch ausgeprägter war dieser Trend bei den technischen Angestellten, bei denen die Entgeltkarriere in aller Regel erst ab der Gehaltsgruppe T4 einsetzte.

Ein gravierender Unterschied zu den gewerblichen Beschäftigten bestand darin, dass es für Angestellte nur eine Entgeltgruppe „unter Eck“, also für un- und angelernte Arbeit, gab, und dass diese zudem schon seit Langem nicht mehr besetzt war. Arbeit unterhalb des Niveaus von Facharbeit, so könnte man daraus schließen, hat es in den kaufmännischen und technischen Angestelltenbereichen der $\mathrm{M}+\mathrm{E}-$ Industrie Baden-Württembergs demnach schon seit Mitte der 1970er Jahre nicht mehr gegeben. Das erscheint nun allerdings recht unwahrscheinlich und deutet eher darauf hin, wie unterschiedlich die Maßstäbe waren und wie wenig ernsthaft die Arbeitsbewertung bei Angestellten lange betrieben wurde. Aussagefähige Arbeitsbeschreibungen gab es selten, eine systematische Arbeitsbewertung fand

\section{TABELLE 1}

Besetzung der Lohn und Gehaltsgruppen in der M+E-Industrie BaWü 1975 bzw. 1977 bis 2005 (Tarifgebiet Nordwürttemberg-Nordbaden)

Angaben in Prozent (gerundet)

\begin{tabular}{|c|c|c|c|c|c|c|c|c|}
\hline \multicolumn{9}{|c|}{ Arbeitswert-/Lohngruppen Arbeiter } \\
\hline & \multicolumn{2}{|c|}{1977} & \multicolumn{2}{|c|}{1985} & \multicolumn{2}{|c|}{1996} & \multicolumn{2}{|c|}{2005} \\
\hline 1 & 1 & \multirow[t]{5}{*}{35} & 0 & \multirow[t]{5}{*}{29} & 0 & \multirow[t]{5}{*}{22} & 0 & \multirow[t]{5}{*}{14} \\
\hline II & 7 & & 4 & & 1 & & 0 & \\
\hline III & 8 & & 8 & & 6 & & 3 & \\
\hline IV & 10 & & 9 & & 7 & & 5 & \\
\hline $\mathrm{V}$ & 10 & & 9 & & 8 & & 6 & \\
\hline $\mathrm{VI}{ }^{*}$ & 11 & \multirow[t]{3}{*}{40} & 10 & \multirow[t]{3}{*}{42} & 9 & \multirow[t]{3}{*}{40} & 8 & \multirow[t]{3}{*}{39} \\
\hline VII & 16 & & 17 & & 14 & & 13 & \\
\hline VIII & 13 & & 15 & & 17 & & 19 & \\
\hline IX & 10 & \multirow[t]{4}{*}{25} & 12 & \multirow[t]{4}{*}{29} & 15 & \multirow[t]{4}{*}{38} & 19 & \multirow[t]{4}{*}{46} \\
\hline$x$ & 6 & & 7 & & 9 & & 10 & \\
\hline $\mathrm{XI}$ & 4 & & 5 & & 6 & & 8 & \\
\hline XII & 4 & & 5 & & 8 & & 10 & \\
\hline
\end{tabular}

Gehaltsgruppen kaufmännische Angestellte

\begin{tabular}{|c|c|c|c|c|c|c|c|c|}
\hline \multirow[b]{2}{*}{ K 1} & \multicolumn{2}{|c|}{1975} & \multicolumn{2}{|c|}{1985} & \multicolumn{2}{|c|}{1996} & \multicolumn{2}{|c|}{2005} \\
\hline & 4 & \multirow[t]{2}{*}{26} & 1 & \multirow[t]{2}{*}{15} & 1 & \multirow[t]{2}{*}{9} & 0 & \multirow[t]{2}{*}{6} \\
\hline K 2* & 22 & & 14 & & 8 & & 6 & \\
\hline K 3 & 21 & \multirow[t]{2}{*}{43} & 22 & \multirow[t]{2}{*}{44} & 20 & \multirow[t]{2}{*}{45} & 17 & \multirow[t]{2}{*}{40} \\
\hline K 4 & 22 & & 22 & & 25 & & 23 & \\
\hline K 5 & 13 & \multirow[t]{3}{*}{32} & 16 & \multirow[t]{3}{*}{40} & 19 & \multirow[t]{3}{*}{47} & 22 & \multirow[t]{3}{*}{53} \\
\hline K 6 & 12 & & 13 & & 15 & & 18 & \\
\hline K 7 & 7 & & 11 & & 13 & & 13 & \\
\hline
\end{tabular}

Gehaltsgruppen technische Angestellte

\begin{tabular}{|c|c|c|c|c|c|c|c|c|}
\hline T1 & 1 & \multirow[t]{2}{*}{7} & 1 & \multirow[t]{2}{*}{5} & 0 & \multirow[t]{2}{*}{2} & 0 & \multirow[t]{2}{*}{1} \\
\hline T $2^{*}$ & 6 & & 4 & & 2 & & 1 & \\
\hline T 3 & 12 & \multirow[t]{2}{*}{32} & 8 & \multirow[t]{2}{*}{24} & 7 & \multirow[t]{2}{*}{22} & 5 & \multirow[t]{2}{*}{19} \\
\hline T 4 & 20 & & 16 & & 15 & & 14 & \\
\hline T 5 & 21 & \multirow[t]{3}{*}{61} & 23 & \multirow[t]{3}{*}{70} & 22 & \multirow[t]{3}{*}{76} & 24 & \multirow[t]{3}{*}{80} \\
\hline T 6 & 23 & & 26 & & 28 & & 30 & \\
\hline T 7 & 17 & & 21 & & 26 & & 25 & \\
\hline
\end{tabular}

* Eckentgeltgruppe $=$ mindestens 3 -jährige Berufsausbildung. Quelle: Südwestmetall (2005).

meist nicht statt, geschweige denn eine, die auf einem analytischen Bewertungssystem beruht hätte.

Wie stellt sich die Entwicklung in der chemischen Industrie dar? Im Grundsatz war die Entwicklungsrichtung dieselbe, d.h. es gab über lange Zeit hinweg einen Trend zu höheren Eingruppierungen, er war jedoch bei den dungsniveau „mehrjährige Berufserfahrung oder eine Fachschulausbildung" gefordert, bei den Kaufleuten fehlte dagegen eine solche Bezugnahme zu einem Bildungsabschluss. In der chemischen Industrie gab es weder bei den technischen noch bei den kaufmännischen Angestellten oberhalb der Einstiegsgruppe für Fachangestellte (K/T 4) eine Deckelung durch die Bezugnahme auf einen als obligatorisch angesehenen Bildungsabschluss.
6 In der betrieblichen Praxis gab es dagegen sowohl vor als auch nach derTarifreform eindeutige Segmentierungen. So kam Kädtler (1991, S. 18) unmittelbar nach Einführung des BETV zum Ergebnis, dass im Segment der Entgeltgruppen 9-12, die tariftechnisch auch ehemaligen Arbeitern offenstehen sollten, keine gewerblichen Arbeitnehmer zu finden waren. Ob sich daran zwischenzeitlich etwas geändert hat, lässt sich mangels neuerer Daten nicht überprüfen. 
TABELLE 2

Besetzung der Lohn- und Gehaltsgruppen in der chemischen Industrie 1960 bzw. 1962 bis 1987

Angaben in Prozent

\begin{tabular}{l|c|c|c|c}
\hline \multicolumn{5}{c}{ Lohngruppen } \\
\hline & 1960 & 1970 & 1980 & 1987 \\
\hline LG I-III & 70 & 68 & 65 & 61 \\
\hline LG IV (Ecklohngruppe) & 30 & 32 & 28 & 31 \\
\hline LG V & 0 & 0 & 7 & 8 \\
\hline & Gehaltsgruppen & & \\
\hline & 1962 & 1970 & 1980 & 1987 \\
\hline K1-K3 & 44 & 36 & 28 & 25 \\
\hline K4 (Eckentgelt, alt K3) & 34 & 35 & 25 & 24 \\
\hline K5-K8 & 22 & 29 & 47 & 51 \\
\hline & & & & \\
\hline T1-T3 & 33 & 26 & 24 & 23 \\
\hline T4 (Eckentgelt, alt T3) & 38 & 35 & 24 & 22 \\
\hline T5-T8 & 29 & 39 & 52 & 55 \\
\hline
\end{tabular}

Quelle: BAVC $(1973,1988)$.

gewerblichen Beschäftigten deutlich schwächer ausgeprägt als in der M+E-Industrie. Zwischen 1955 und 1987 und damit in einem ähnlich langen Zeitraum wie wir ihn zuvor in der $\mathrm{M}+\mathrm{E}$-Industrie analysiert haben, erhöhte sich bei den Gewerblichen der Anteil der Beschäftigten in der Ecklohngruppe und darüber lediglich um 8 Prozentpunkte von 31 auf $39 \%^{7}$ (Tabelle 2). Der Vergleich zur M+E-Industrie zeigt auch: Un- und angelernte Arbeit war in der chemischen Industrie lange Zeit wesentlich bedeutsamer als in der M + E-Industrie. Die Quote der Geringqualifizierten unter den Arbeitern lag 1987, dem Zeitpunkt der Tarifreform in der chemischen Industrie, bei fast $70 \%$, in der $\mathrm{M}+\mathrm{E}$-Industrie BaWü war sie schon auf ca. $25 \%$ gesunken. Dementsprechend war die Entgeltpolitik der chemischen Industrie im gewerblichen Bereich wesentlich stärker auf die Geringqualifizierten ausgerichtet.

Bei den Angestellten, die zum Zeitpunkt der Tarifreform 1987/88 einen Anteil von $48 \%$ an den Beschäftigten hatten (M+E-Industrie $33 \%)$, war der Trend zur Höhergruppierung ähnlich stark ausgeprägt wie in der M+E-Industrie. Zwischen 1962 und 1987 stiegen die Beschäftigtenanteile, die in die obersten vier Entgeltgruppen eingruppiert waren (K/T 5-8), bei den Kaufleuten um 29 und bei den Technikern um 26 Prozentpunkte an. Ein gravierender Unterschied zur M +E-Industrie bestand allerdings darin, dass bei Abschluss des BETV (1987) ein Viertel der Angestellten in Gehaltsgruppen für Angelernte eingruppiert war (K/T 1 -3). 1962 lag der Anteil sogar noch deutlich höher. Anders gesagt: Un- und angelernte Arbeit war in der chemischen Industrie nicht nur bei Arbeitern, sondern auch bei Angestellten erheblich bedeutsamer als in der M+E-Industrie. ${ }^{8}$

\subsection{Effekte der Tarifreformprojekte}

Welche Effekte hatten die Entgeltreformprojekte speziell für die Bewertung und Bezahlung von Facharbeit? Will man diese Frage beantworten, sind zwei Wirkungsebenen zu unterscheiden: die tarifsystematische und die effektive. Tarifsystematisch im Rang deutlich nach oben rückten in der $\mathrm{M}+\mathrm{E}$-Industrie z. B. qualifizierte Arbeitsaufgaben in der Produktion und in ihrem Umfeld, etwa die Vorarbeiter, die Betriebselektriker, die Montageanlagenführer und die Maschineneinrichter. Kleinere Ranggewinne gab es bei den Meistern und den Werkstattprogrammierern. Tarifsystematisch im Rang nach unten korrigiert wurden Sekretärinnen, NC-Programmierer, Einkaufssachbearbeiter, technische Zeichner, Rechnungsprüfer sowie Maschinenbediener, Gießer, Maschinenbeschicker, Montierer, Schweißer und Bürohilfen.

Die Komplikation besteht nun darin, dass die tarifsystematisch intendierten Wirkungen von den effektiven abweichen. Ursächlich hierfür war, dass sich die tatsächlichen Eingruppierungen, die Verrechnungsgrade im Leistungsentgelt und die Vergütung von Belastungen über die Jahre hinweg von der tariflichen Papierlage deutlich entfernt hatten. Zum einen schlugen sich die Dauer der Betriebszugehörigkeit, die Loyalität und auch die Leistung (tarifwidrig) in einer zu hohen Eingruppierung nieder. Nicht zu vergessen ist das Engagement der Betriebsräte, die für ihnen besonders nahestehende und auch kampfstarke Beschäftigtengruppen eine höhere, nicht unbedingt tarifkonforme Eingruppierung durchsetzen konnten. Gleiches gilt für das Engagement von Führungskräften für ihnen wichtige Personen bzw. Beschäftigtengruppen. Kurzum, es gab vielfach „politische“ Eingruppierungen und damit eine verdeckte Entgeltspanne zwischen realem und tarifvertraglich tatsächlich begründbarem Verdienst. An der Aufdeckung dieser Spanne waren über lange Zeit weder die Betriebs- noch die Tarifparteien interessiert. Im Zuge der ERA-Einführung kamen nun aber die historisch entstandenen Verwerfungen ans Licht, mit der Folge, dass aus tarifsystematischen „Gewinnern“ von ERA real „Verlierer“"werden konnten.

Welche realen Effekte haben sich nun ergeben? Mit Blick auf die tatsächlichen Auf- und Abwertungen von Arbeitsaufgaben zeigen unsere Befragungsergebnisse für die $\mathrm{M}+\mathrm{E}$ -

7 Oberhalb der Ecklohngruppe wurde erst 1973 eine zusätzliche Entgeltgruppe für "Spezialisten“, die LG V, eingeführt und dies parallel zu einer Aufspreizung der Lohngruppen I und II.

8 Nach Darstellung von Brocker (2011, S. 180), dem vormaligen Hauptgeschäftsführer von Gesamtmetall, haben sich zwischenzeitlich die Qualifikationsunterschiede zwischen beiden Branchen eingeebnet. Rund $65 \%$ der $\mathrm{M}+\mathrm{E}-\mathrm{Be}-$ schäftigten sind demnach ausgebildete Facharbeiter (chemische Industrie $62 \%$ ), weitere $16 \%$ sind Akademiker (chemische Industrie $18 \%$ ) und beide Branchen hatten eine vergleichbare Quote von Auszubildenden (ca. $6 \%$ ). 
Industrie BaWü ${ }^{9}$, dass die intendierte Höherbewertung der Facharbeit in der Produktion und in produktionsnahen Bereichen in einem Großteil der Betriebe tatsächlich erreicht wurde. Sofern die höher bewerteten Facharbeitertätigkeiten spezifiziert wurden, handelt es sich vornehmlich um solche im Werkzeugbau, in der Instandhaltung, um (Werkstatt)Programmierer, Einrichter, Anlagenführer und junge Facharbeiter. Teilweise profitieren auch qualifizierte technische Angestellte, insbesondere (Entwicklungs)Ingenieure, Konstrukteure und qualifizierte Techniker. Ähnlich häufig erfahren untere bzw. mittlere Führungskräfte eine Aufwertung (in $20 \%$ der Betriebe), etwa Vorarbeiter, Gruppenführer, Meister oder Schichtführer. Zu höheren Bewertungen von Un- und Angelernten kam es dagegen fast nirgends. Im Durchschnitt wurden Verdienste der ERA-Gewinner um $163 €$ pro Monat erhöht, in Einzelfällen wurden Beträge bis $1.500 €$ erreicht (vgl. Bahnmüller/ Schmidt 2009, S. 232).

Den Aufwertungen bei den einen stehen allerdings Abwertungen bei anderen gegenüber. Neben Beschäftigten in Sekretariatsfunktionen, Kaufleuten und Technikern waren davon häufig Angelernte in der Produktion (44\% der Betriebe) betroffen. Aber auch im Segment der Facharbeit kam es nicht nur zu Auf-, sondern auch zu Abwertungen. Betroffen hiervon waren Beschäftigte, die bisher als Facharbeiter galten und so auch bezahlt wurden. So waren nicht wenige Beschäftigte etwa in der Automobilmontage in EG 7 oder höher eingruppiert und sollten nun in die EG 6 oder auch EG 5 abgruppiert werden. Dieser „Streit um Facharbeit" war allerdings mit der Auffächerung der Berufsabschlüsse im ERA nicht verkoppelt. Im Kern ging es dabei vielmehr um die Frage, ob eine nicht unbedingt tarifkonforme, sondern „politische" Arbeitsbewertung Bestand hat, wie sie Beschäftigte und Betriebsräte über die Jahre realisieren konnten.

Dennoch kamen die von uns befragten Betriebsräte der M+E-Industrie BaWü unmittelbar nach Ende des offiziellen Einführungszeitraums von ERA in ihrer Gesamtbewertung mehrheitlich zu der Einschätzung, die intendierten (formalen) Gleichbehandlungs- und Gerechtigkeitsziele seien im Wesentlichen erreicht worden (Gleichbehandlung von Arbeitern und Angestellten in der Bewertung von Arbeit, Leistung und Belastungen). Auch die Aufwertung von Facharbeit als Ergebnis der Gleichbehandlung gehört dazu. Dagegen stehen jedoch Entgelteinbußen, die primär Neubeschäftigte treffen, welche nicht von den Entgeltabsicherungen profitieren, die für die „Altbelegschaften“ gelten. Mehr als die Hälfte der Beschäftigten musste diese Entgeltabsicherung in Anspruch nehmen, da ihr bisheriger Verdienst über dem neuen lag. Eine Mehrheit der Betriebsräte fürchtete auch eine dauerhafte Absenkung des betrieblichen Entgeltniveaus und knapp die Hälfte konstatierte zudem Entgeltverluste der Stammbelegschaften, da bei Beschäftigten, die vor ERA mehr verdient hatten als danach, maximal $10 \%$ ihres letzten Bruttoentgelts mit künftigen Entgeltsteigerungen verrechnet werden konnten.
Die Effekte des BETV in der chemischen Industrie sind mit jenen des ERA in der M + E-Industrie insofern vergleichbar, als nunmehr auch hier ein Eingruppierungsschema vorliegt, das auf Basis einheitlicher Tätigkeitsmerkmale und Entgeltgruppenbeschreibungen Arbeiter und Angestellte entsprechend der erforderlichen Qualifikationen und der Freiheitsgrade in der Arbeit einer Entgeltgruppe zuordnet. Sofern systemkonform verfahren wird, kommen damit reine Statusunterschiede bei der Eingruppierung und damit der Bezahlung nicht mehr zum Tragen. Profitiert haben davon auch hier vor allem Facharbeiter und Handwerker, deren Bezahlung sich laut Kädtler (1991, S. 19) deutlich und dauerhaft verbessert hat. Auch überlappt sich die Endstufe der EG 8 mit den Anfangsstufen der beiden nachfolgenden Entgeltgruppen, wodurch entgeltsystematisch eine Öffnung der bisher den Angestellten vorbehaltenen EG 9 und höherer Entgeltgruppen materiell gut fundiert ist. ${ }^{10}$

Gleichwohl ist hierdurch laut Kädtler (1991) de facto kein für alle Beschäftigtengruppen gleichermaßen durchlässiges und in diesem Sinne auch wirklich einheitliches Entgeltsystem entstanden. Die Eingruppierung von Arbeitern und Angestellten erfolge nun zwar nach gleichen Grundsätzen, jedoch verharrten die qualifizierten gewerblichen Beschäftigten noch immer in den EG 6 bis EG 8. Die EG 6 bedeute für Gewerbliche und ehemalige Angestellte nach wie vor Unterschiedliches. Die gewerblichen Fachkräfte blieben hier stehen oder stiegen allenfalls in die EG 7, selten in die 8 auf, für Angestellte sei die EG 6 dagegen „Startplatz und der Bereich bis EG 8 Einlaufphase einer Laufbahn, die im Übrigen im tariflichen Oberhaus ab EG 9 absolviert wird“ (Kädtler 1991, S. 31). Allerdings berichtet Kädtler auch von Betriebsfällen, in denen relevante Anteile der Gewerblichen, die im Altsystem in der höchsten Lohngruppe eingruppiert waren, nunmehr in die EG 9 eingestuft wurden, was vorher nicht denkbar war. Wie bedeutsam diese Beispiele sind und ob sie in den Jahren nach Abschluss der Untersuchung von Kädtler Nachahmer fanden, kann mangels aktueller empirischer Untersuchungen, mit denen sich die individuellen oder gruppenspezifischen Wanderbewegungen erfassen lassen, nicht gesagt werden. Eindeutig zu den Verlierern der Tarifreform zählen Kädtler

9 Die hier präsentierten empirischen Befunde basieren auf einer 2007/2008 durchgeführten, standardisierten, schriftlichen Befragung von Betriebsräten aller tarifgebundenen Betriebe der $\mathrm{M}+\mathrm{E}-$ Industrie Baden-Württembergs. Präsentiert werden hier ausschließlich die Befragungsergebnisse für jene Betriebe, die ERA zum Zeitpunkt der Befragung bereits eingeführt hatten ( $N=149$ ). Sie repräsentieren $14 \%$ aller tarifgebundenen Betriebe der $\mathrm{M}+\mathrm{E}$-Industrie BadenWürttembergs, in denen $43 \%$ aller vom ERA betroffenen Beschäftigten tätig sind (insgesamt 283.092) (siehe Bahnmüller/Schmidt 2009, S. 43ff.).

10 Die Endstufe der EG 8 liegt nach Auskunft des WSI-Tarifarchivs derzeit im Geldbetrag über der Eingangsstufe der EG 10. 
TABELLE 3

Entgeltgruppenbesetzung in der chemischen Industrie 1988 bis 2010

Angaben in Prozent

\begin{tabular}{lc|c|c|c|c|c|c|c}
\hline & 1988 & 1989 & 1991 & 1993 & 1996 & 2006 & \multicolumn{2}{|c|}{2010} \\
\hline & West & West & West & West & West & West & West & Gesamt \\
\hline EG 1-3 & 21 & 22 & 21 & 21 & 15 & 9 & 9 & 9 \\
\hline EG 4-5 & 18 & 17 & 17 & 17 & 17 & 12 & 11 & 11 \\
\hline EG 6*-8 & 30 & 31 & 32 & 32 & 36 & 39 & 40 & 40 \\
\hline EG 9-13 & 31 & 30 & 30 & 29 & 32 & 40 & 40 & 40 \\
\hline
\end{tabular}

* EG 6 Eckentgeltgruppe $=$ mind. 3-jährige Berufsausbildung.

Quelle: BAVC $(2007,2010)$
Auf mittlere Frist geht der Arbeitgeberverband gleichwohl von einer weiteren Erhöhung der Facharbeiter- bzw. des Anteils höher qualifizierter Tätigkeiten aus (BAVC 2010). Umstritten ist, ob davon vor allem die akademischen Berufe profitieren werden und im Gegenzug die Kerngruppen des deutschen Produktionsmodells, die industriellen Fachkräfte mit berufsqualifizierendem Abschluss, ins Hintertreffen geraten sind. Eine jüngst abgeschlossene Studie zu den Perspektiven von Chemielaboranten gibt jedoch Entwarnung. Ihre Zahl ist in den letzten zehn Jahren gestiegen, auch die Ausbildungszahlen nahmen überkonjunkturell zu, und zudem zeigen sich auch die Personalmanager und operativen Führungskräfte der Branche als überzeugte Verfechter der dualen Ausbildung mit deutlicher Reserve gegenüber dem Bachelorabschluss (Kädtler/Neumann 2012). Die berufliche Position von Laboranten in der chemischen Industrie scheint demnach ausgesprochen stabil. Gezählt sind dagegen die Tage von Laborwerkern und Beschäftigten im Labor ohne einschlägigen Berufsabschluss (ebd.).

In der M+E-Industrie BaWü hat die einheitliche Arbeitsbewertung von Arbeitern und Angestellten zunächst zu einer deutlichen Erhöhung des Anteils der Beschäftigten geführt, die unterhalb der Eckentgeltgruppe eingruppiert sind. Er hat sich von $17 \%$ auf $33 \%$ erhöht. Gründe für den drastischen Anstieg dürften auf mehreren Ebenen zu suchen sein. Erstens wurden durch die komplette Neubewertung aller Arbeitsaufgaben auf Basis eines strikt anforderungsorientierten Systems alle nicht tarifkonformen Eingruppierungen korrigiert, was, wie dargestellt, bei Anlerntätigkeiten und im Übergang zu Facharbeit zu deutlich mehr Ab-als Aufwertungen führte. Zweitens sind Belastungen, die bisher im summarischen System bei Arbeitern zu höheren Eingruppierungen führen konnten, von der Grundentgeltfindung abgekoppelt und in eine von der Entgeltgruppe unabhängige Zulage überführt, was den Anteil unterhalb der Eckentgeltgruppe ebenfalls ansteigen ließ. Drittens wurden nun auch Angestelltentätigkeiten einer systematischen Arbeitsbewertung unterzogen, was zur Folge hatte, dass in kaufmännischen und administrativen Bereichen erstmals in relevanter Anzahl Anlerntätigkeiten ausgewiesen sind. Viertens sind nach Einführung des ERA die Eingruppierungsdaten der drei bisher getrennten Tarifgebiete BadenWürttembergs zusammengefasst, und schließlich haben fünftens die Arbeitgeber, angeleitet durch Südwestmetall, ERA ausgesprochen restriktiv angewendet (vgl. Bahnmüller/ Schmidt 2009).

Im weiteren Fortgang nach Einführung von ERA ist der Anteil „unterhalb des Facharbeiterecklohns anfangs von $33 \%$ (2008) auf $30 \%$ (2009) gesunken, um seither auf diesem Niveau zu verharren. Auch im oberen Segment der Entlohnung (EG 13-17) gibt es kaum Bewegung. Nach einer kleineren Korrektur von 24 auf $26 \%$ in den Jahren 2008/2009 verbleibt der Anteil seither auf diesem Niveau. Analoges gilt für das Segment der Facharbeit (EG 7-12). Dieses ist mit von anfangs $43 \%$ auf nunmehr $45 \%$ nur leicht gestiegen (jeweils 2008 - 2012). Die Zeitreihe umfasst zwar 
erst fünf Jahre, es gibt bisher jedoch keine Anzeichen dafür, dass sich die Höhergruppierungsdynamik vor ERA fortsetzt. Seit 2008 sind die Entgeltgruppenbesetzungen jedenfalls weitgehend stabil und die Arbeitgeberseite setzt auch Einiges daran, dass dies so bleibt.

\section{Fazit}

Die Neubewertung von gewerblicher Facharbeit, ihre Aufwertung und finanzielle Gleichstellung mit technischen und kaufmännischen Tätigkeiten, war eines der zentralen Ziele der Entgeltreformprojekte, die vor nunmehr 25 Jahren in der chemischen Industrie und vor zehn Jahren in der M+E-Industrie ins Werk gesetzt wurden. Hierdurch wurde nicht nur eine Störvariable in der Kooperation von Arbeitern und Angestellten beseitigt, sondern die beruflichen Aufstiegs- und Entwicklungsmöglichkeiten, insbesondere für qualifizierte gewerbliche Facharbeit wurden verbessert. $\mathrm{Ob}$ die dadurch gesetzten monetären Anreize ausreichen, um die absehbaren Rekrutierungsprobleme von Fachkräften zu lösen, muss hier offenbleiben. Die Tarifparteien haben jedoch Zeichen für die Bedeutsamkeit gesetzt, die sie Beschäftigten mit dualer Ausbildung auch perspektivisch zuweisen. Profitiert hat von den Reformprojekten vor allem gewerbliche, teilweise auch kaufmännische und technische Facharbeit. Qualifizierte gewerbliche Facharbeit ist im Rang vielfach nach oben gehoben worden, es sind Entgeltsteigerungen eingetreten und tarifsystematisch sind die beruflichen und monetären Entwicklungsmöglichkeiten durch eine durchlässige und einheitliche Gestaltung der Eingruppierungsschemata verbessert worden. Durch ihre anforderungsbezogene Logik kennen die reformierten Tarifverträge grundsätzlich auch keine an formalen Bildungsabschlüssen festgemachten Zugangsbeschränkungen für bestimmte Funktionen bzw. Positionen. Die Tarifparteien waren insofern den Bildungspolitikern voraus. Sie hatten Durchlässigkeit und Gleichwertigkeit formal und non-formal erworbener Kompetenzen bereits zu einem Prinzip von Entgeltsystemen gemacht, als in der Bildungspolitik noch versucht wurde, den Zugang zu (Aus)Bildungseinrichtungen an formale Bildungsabschlüsse zu binden. Bezeichnenderweise dienten die im ERA-BaWü zugrunde gelegten Merkmale der Arbeitsbewertung und deren Stufung als Bezugspunkte bei der Entwicklung des Deutschen Qualifikationsrahmens (siehe den Beitrag von Nehls in diesem Heft).

Dass trotz der grundsätzlichen Durchlässigkeit der tariflichen Entgeltsysteme in der betrieblichen Praxis weiterhin Segmentierungslinien entlang der bisherigen Statusgrenzen und formaler Ausbildungsabschlüsse existieren, ist wahrscheinlich, steht jedoch auf einem anderen Blatt. Es ist nicht den Tarifverträgen anzulasten, sondern den betrieb- lichen Interessen- und Machtkonstellationen, innerhalb derer sie ausgestaltet und angewandt werden. Kädtler (1991) hat unmittelbar nach Einführung des BETV in der chemischen Industrie Hinweise auf die Persistenz der alten Demarkationslinien geliefert. Es wäre an der Zeit, diese Befunde zu aktualisieren und auch für die M+E-Industrie zu überprüfen - zumal einiges dafür spricht, dass auch dort die alten Statusunterschiede und Zugangsbeschränkungen fortwirken. An deren Aufrechterhaltung dürften auch nicht nur Personalmanager interessiert sein, die ein „Durchsteigen“ ehemaliger Arbeiter in die traditionellen Angestelltenregionen und eine dadurch ausgelöste Höhergruppierungsdynamik fürchten, sondern auch Beschäftigtengruppen, die ihre privilegierten Zugänge zu gut dotierten Arbeitsplätzen gefährdet sehen.

Die Beseitigung der Entwicklungsblockaden und die Aufwertung von (gewerblicher) Facharbeit sind gleichwohl nicht nur auf dem Papier, sondern in den meisten Betrieben auch real erfolgt. Dennoch gibt es in der M+E-Industrie auch Beispiele von Facharbeitstätigkeiten, die abgewertet wurden. Die hauptsächlichen Verlierergruppen sind jedoch Beschäftigte in Anlerntätigkeiten der Produktion und auch solche in weniger komplexen kaufmännischen, technischen, Verwaltungs- und Sekretariatsfunktionen. Durch tarifliche Absicherungsmaßnahmen wurden zwar die bisherigen Verdienste der „Altbelegschaften“ weitgehend abgesichert, die Lasten mussten und müssen allerdings die nachrückenden Neubelegschaftsmitglieder tragen, bei denen die niedrigeren Eingruppierungen mancher Arbeitsaufgaben erst entgeltwirksam werden.

$\mathrm{Zu}$ den problematischen Seiten der Entgeltreformprojekte gehört zudem, dass parallel zur tarifsystematischen und vielfach auch effektiven Aufwertung von Facharbeit das Anforderungsniveau abgesenkt wurde, ab dem von Facharbeit gesprochen werden kann. Zur dreijährigen ist die zweijährige Berufsausbildung hinzugekommen. Praktisch bedeutsam ist Letztere in den Betrieben bisher nicht geworden, es sind damit jedoch Möglichkeiten geschaffen worden, Druck auf das Eingruppierungsniveau, insbesondere im Grenzbereich von gelernter und ungelernter Arbeit, auszuüben und es nach unten zu korrigieren.

Ein weiterer Preis der Entgeltreformprojekte scheint schließlich eine verlangsamte, wenn nicht ausgesetzte Dynamik der Höhergruppierung zu sein. Diese war vor den Entgeltreformprojekten wesentlich stärker ausgeprägt als danach. Und auch der Trend war eindeutig: Der Anteil der Beschäftigten in Anlerntätigkeiten nahm kontinuierlich ab, jener in qualifizierten Tätigkeiten, in denen mindestens eine abgeschlossene dreijährige Berufsausbildung vorausgesetzt wird, nahm zu. Damit scheint es nun vorbei zu sein. In der $\mathrm{M}+\mathrm{E}$-Industrie BaWü sind seit der ERA-Einführung die Anteile der drei Segmente für Anlerntätigkeiten, für Facharbeit sowie für Tätigkeiten in den oberen Eingruppierungsgruppen weitgehend stabil. Auch in der chemischen Industrie hat sich seit 2006 nichts mehr bewegt. Die betriebliche Eingruppierungspolitik tritt auf der Stelle. Die Hoff- 
nung der Gewerkschaften, dass auf Basis der modernisierten und aktualisierten Entgeltrahmentarifverträge eine aktive und offensive Eingruppierungspolitik der Betriebsräte wieder Platz greifen könnte, scheint sich jedenfalls nicht zu erfüllen.

\section{LITERATUR}

Bahnmüller, R./Schmidt, W. (2009): Riskante Modernisierung des Tarifsystems. Die Reform des Entgeltrahmenabkommens in der Metall- und Elektroindustrie Baden-Württembergs, Berlin

Brocker, U. (2011): Chemische Industrie und Metall- und Elektroindustrie.

Unterschiede und Gemeinsamkeiten, in: Vorscherau, E./Vassiliadis, M. (Hrsg.): Die Frey'sche Formel - Stationen der Sozialpartnerschaft. Festschrift zu Ehren von Hans Paul Frey, Wiesbaden, S. 178-187

Brunkhorst, Ch./Burkhard, O./Scherbaum, M. (Hrsg.) (2006): Eine neue AERA Tarifverträge für die Zukunft, Hamburg

Bundesarbeitgeberverband Chemie (BAVC) (1973/1988): Zahlen zur Sozialpolitik in der chemischen Industrie, Wiesbaden

Bundesarbeitgeberverband Chemie (BAVC) (2007): Trend zu höher qualifizierten Arbeitsplätzen, in: Informationsbrief (8), S. 4-5

Bundesarbeitgeberverband Chemie (BAVC) (2010): Mehr qualifizierte Arbeitsplätze, in: Informationsrundbrief (8), S. 4-5

Bundesentgelttarifvertrag für die chemische Industrie (BETV) (1987): http://www.boeckler.de/pdf/ta_tvg60 1987_chemie_bundesentgelttarifvertrag.pdf (letzter Zugriff: 02.01.2013)

Ehlscheid, C./Meine, H./Ohl, K. (Hrsg.) (2006): Handbuch Arbeit, Entgelt, Leistung. Tarifanwendung im Betrieb, Frankfurt a. M.
IG Metall BaWÜ/Bildungsstätte Lohr (Hrsg.) (2004): ERA Handbuch Wissen I. Arbeitsbewertung und Belastungsbewertung, Lohr

Kädtler, J. (1991): Der Bundesentgelttarifvertrag für die chemische Industrie Leitbild einer neuen Tarifpolitik? Berliner Arbeitshefte und Berichte zur sozialwissenschaftlichen Forschung (43), Berlin

Kädtler, J. (2002): Das schiedlich-friedliche Jahrhundertwerk, in: Express. Zeitschrift für sozialistische Betriebs- und Gewerkschaftsarbeit (4)

http://labournet.de/branchen/chemie/allg/kaedtler.html

Kädtler, J./Neumann, U. (2012): Industrielle Fachkräfte unter Druck? Das Beispiel der Laborantinnen und Laboranten in der Chemie- und Pharmaindustrie: Hans-Böckler-Stiftung, Arbeitspapier (257), Düsseldorf

Meine, H./Ohl, K./Rohner, R. (Hrsg.) (2011): Handbuch Arbeit, Entgelt, Leistung. Entgeltrahmentarifverträge im Betrieb, Frankfurt a. M.

Südwestmetall (2005): Lohn- und Gehaltserhebung April 2005 Nordwürttemberg/Nordbaden, Stuttgart

AUTOR

REINHARD BAHNMÜLLER, Dr., Forschungsinstitut für Arbeit, Technik und Kultur (F. A.T. K.) an der UniversitätTübingen. Arbeitsschwerpunkte: Arbeits- und Industriesoziologie, Industrielle Beziehungen.

reinhard.bahnmueller@uni-tuebingen.de 